\title{
Mapping the Intrinsic Stochasticity of Tokamak Divertor Configuration
}

\author{
S. R. Barocio, E. Chávez-Alarcón, and C. Gutierrez-Tapia \\ Instituto Nacional de Investigaciones Nucleares, Physics Department, A.P.18-1027, 11801 México D.F.
}

Received on 21 March, 2005. Revised version received on 13 February, 2006

\begin{abstract}
Poloidal divertors are, more than ever before, a crucial topic for the advancement of magnetic fusion technology. Due to the often non linear and stochastic nature of the plasma edge phenomena, canonical mapping has provided a powerful method at modelling their characteristics, albeit many authors rely on heuristically adapted schemes. Thus, it is reported here a specific and physically consistent map model of the tokamak single null magnetic configuration, assuming plasma-field equilibrium, based on the construction of a fundamental Hamiltonian form. Then, the magnetohydrodynamically non ideal perturbations are introduced through the Rayleigh function of the system. As an illustration, the resulting compact canonical map is applied to the analysis of some of the most relevant features of the edge magnetic topology.
\end{abstract}

Keywords: Tokamak; Hamiltonian models; Canonical mapping

\section{INTRODUCTION}

Poloidal ergodic divertors constitute a prime technique in the management of impurities [1], magnetohydrodynamic (MHD) stabilization [2] and high performance mode inducement [3] for present day and future tokamaks. In particular, the challenge posed by the heat flux impact on the vessel target inner surfaces has been considerably averted by means of divertor dissipative techniques [4]. In an MHD ideal tokamak the plasma is confined to a region of nested toroidal magnetic surfaces limited by one separatrix surface from the outer surfaces that divert the plasma exhaust to the target. In real near integrable tokamaks, marginal resistive reconnection, error magnetic asymmetries, etc., transform the separatrix into a stochastic layer of semi-open field lines. These evolve to a rich and complex homoclinic topology [5] on which the patterns of deposition on the target depend. It has been long known that magnetic field lines follow the trajectories of a $1 \frac{1}{2}$ degree of freedom Hamiltonian [6] and where parallel conductivity largely outweighs transversal diffusion, so that plasma flows preferentially along the magnetic field lines. Therefore, the inherent use of area preserving maps to model the system has turned out to be an often revealing as well as processing-time saving practice since as early as the 1950's [7, 8]. Built upon them, the so called test-particle theories began exploring transference phenomena in stochastic field equilibria [9] by exploiting the vast possibilities of these discrete maps $[10,11]$ and even modelling actual tokamak facilities [12].

Nevertheless, a word of caution should be said about the geometry selected to represent tokamak equilibria, as merely adapting already existing maps seems to be a rather common practice, according to which, one produces a map 'ab initio and check its relevance a posteriori [13]. It is, indeed, true that finding a map exactly equivalent to the field line equations is not necessarily trivial. Yet, some particular adaptations involve considerable risks. To start with, the well known (Chirikov-Taylor) Standard map [14] has proven not to be a compatible model for a tokamak $[13,15]$. It can also be shown, for instance. That slab geometries, despite their popularity, are not suited for describing fluxes as plasma current density in slab magnetic islands grows with their width, in contradiction with the actual behaviour where stochasticity is precisely enhanced by cooling [16]. Even the, perhaps, most consistent archetypal tokamak map, the one parameter single null divertor map [17] remains disconnected from most of the physical significance of its variables and parameters. It is even affirmed that this map cannot address a particular device [18]. Furthermore, it has been unnecessarily composed for no other reason than making it look aesthetically appealing although it may have no physical significance [11]. In contrast with previous attempts by other authors, the aim of the present work is to construct a specific comprehensive, and yet compact, hamiltonian mapping model of the tokamak poloidal divertor on a physically meaningful basis in order to gain a better understanding of some of the often misinterpreted tokamak single null edge phenomena.

The contents are organised as follows: in Section 2 an archetypal Hamiltonian of the single null tokamak divertor configuration is developed so to satisfy the Hamilton equations of the equilibrium magnetic field lines in generalised coordinates. The equations are solved, first conventionally, for the ideal MHD axisymmetric case and then, in Section 3 , by constructing a more realistic near integrable difference equation scheme based on the concept of constraint relaxation. This scheme is expressed, by more than one method, in the form of a canonical map whose parameters are adjusted on typical operational values such as those of COMPASS-D tokamak (major radius: $R=0.557 \mathrm{~m}$, minor horizontal semi-axis: $a=0.232 \mathrm{~m}$, minor vertical semi-axis: $b=0.385 \mathrm{~m}$, aspect ratio: $R / a=2.53$, material: Inconel 625 , maximum toroidal field on axis: $2.1 \mathrm{~T}$, rise time: $\sim 1 \mathrm{~s}$, flat top duration at top field: $1 \mathrm{~s}$, decay time $\sim 1 \mathrm{~s}$, number of field coils: 16 , field ripple on axis $<0.006$, plasma current: $<400 \mathrm{kA}$, plasma duration without current drive: $\sim 600 \mathrm{~ms}$, plasma rise time: $<80 \mathrm{~ms}$, maximum vertical field on axis: $200 \mathrm{mT}$ ) $[19,20]$, as a reference. Section 4 presents an application of the model to the study of edge field structure, on a stability criterion. A discussion of some of the implications of such a structure for the transport is included and the resonant-like origin of the deposition patterns on the target is predicted. Conclusions and some final remarks give form to Section 5 . 


\section{THE IDEAL SINGLE NULL TOKAMAK CONFIGURATION}

We consider initially a cylinder coordinate frame $(R, \varphi, Z)$ where $Z=0$ describes the tokamak midplane, $R=0$ coincides with the toroidal axis (often known as central line) while $\varphi$ is the conventional toroidal angle. On the assumption of an MHD ideal plasma-magnetic field equilibrium satisfying the Grad Shafranov equation $[12,21]$, namely, where non resistive Maxwell equations apply [22], one has that, in particular, $\nabla \cdot \mathbf{B}=0$ and therefore the usual vector potential $\mathbf{A}$ exists such that $\mathbf{B}=\nabla \times \mathbf{A}$ Then, given the metric condition $\mathbf{B} \cdot \hat{R} / d R=\mathbf{B} \cdot \hat{\varphi} / R d \varphi=\mathbf{B} \cdot \hat{Z} / d Z$ where the unitary vector $\hat{\varphi}=R \nabla \varphi$, then a magnetic field line evolves along the toroidal direction as

$$
\frac{d R}{d \varphi}=-\frac{1}{\mathbf{B} \cdot \hat{\varphi}} \frac{\partial R \mathbf{A} \cdot \hat{\varphi}}{\partial Z}, \quad \frac{d Z}{d \varphi}=\frac{1}{\mathbf{B} \cdot \hat{\varphi}} \frac{\partial R \mathbf{A} \cdot \hat{\varphi}}{\partial R}
$$

Always in the MKSA unit system and provided that a tokamak toroidal field is $\mathbf{B} \cdot \hat{\varphi}=B_{0} R_{0} / R$ where the constant $B_{0}=$ $\mu_{0} \kappa I_{C} / 2 \pi R_{0}$, the magnetic permeability is $\mu_{0}$, the mean major radius $R_{0}$, the effective toroidal field coil current $I_{C}$ and the number of turns $\kappa$, we define our set of generalised coordinates $(\rho, z, \tau)$ with

$$
\rho \equiv \ln \left(R / R_{0}\right)
$$

so to obtain respectively from equations (1) that

$$
\frac{\partial \mathcal{H}}{\partial z}=-\frac{d \rho}{d \tau}, \quad \frac{\partial \mathcal{H}}{\partial \rho}=\frac{d z}{d \tau}
$$

with the Hamiltonian functional

$\mathcal{H}[\rho, z ; A \varphi(\rho, z, \tau)] \equiv \frac{2 \pi}{\kappa B_{0}} e^{\rho} A_{\varphi}\left(R=R_{0} e^{\rho}, z=Z / u_{0}, \varphi=2 \pi \tau / \kappa\right)$

where $u_{0}$ is the length unit and $A_{\varphi} \equiv \mathbf{A} \cdot \hat{\varphi}$ so that $\mathcal{H}=$ $\psi_{P} / \kappa B_{0} R_{0}, \psi_{P}$ being the poloidal flux function.

We proceed now to design an $\mathcal{H}=H(\rho, z)$ form for the single null case so that all the fundamental properties of this configuration be reproduced with a minimum number of parameters. In point of fact, it is well known that the correlation dimensionality of equilibrium field fluctuations measured in a tokamak suggest that this number of parameters is close to three [23]. Thus, let us require that a frame of reference exists where a toroidally symmetrical $H(\rho, z)$ can be expressed as an expansion around $\rho=0, z=0$ with no mixed terms so to preserve its separability, namely,

$$
H(\rho, z)=\sum_{j \geq 0} \beta_{j} \rho^{\prime}+\sum_{j \geq 0} \gamma_{j} z^{j}
$$

where $\beta_{j}, \gamma_{j}$ are constants for some particular value $\alpha$ of the rotation matrix

$$
\mathbf{R}=\left(\begin{array}{cc}
\cos \alpha & -\sin \alpha \\
\sin \alpha & \cos \alpha
\end{array}\right)
$$

so that

$$
\left(\begin{array}{c}
\rho \prime \\
z^{\prime}
\end{array}\right)=\mathbf{R}\left(\begin{array}{l}
\rho \\
z
\end{array}\right)
$$

We can now impose the following four constraints on the $\rho$ ' vs $z$ plane. In the first place that any horizontal line cut a magnetic surface in no more than two points, that is, $\beta_{j}=$ 0 for $j \geq 3$. Secondly, that any vertical line cut a magnetic surface in no more than three points, or, $\gamma_{j}=0$ for $j \geq 4$. Likewise, that the plasma column be centered on the magnetic axis whereby the value of $H$ is an extremal there and therefore $\beta_{1}=0$. Finally, that the magnetic axis lie on the midplane so that the magnetic surfaces are symmetrical in $z^{\prime}$ as $z^{\prime} \longrightarrow 0$ and hence $\gamma_{1}=0$. We arrive, then, at the autonomous Hamiltonian

$$
H\left(x \prime, z^{\prime}\right)=\beta_{2} \ln ^{2}\left(1+x \prime / R_{0}\right)+\gamma_{2} z^{2}+\gamma_{3} z^{\prime}
$$

where, if $\alpha$ is the angle between the $z-$ and $z$-axes, as it will be seen in Fig. 1 for later purposes. Then

$$
\begin{gathered}
x \prime(\rho, z)=R_{0}[\exp (\rho \cos \alpha-z \sin \alpha)-1] \\
z \prime(\rho, z)=\rho \sin \alpha+z \cos \alpha .
\end{gathered}
$$

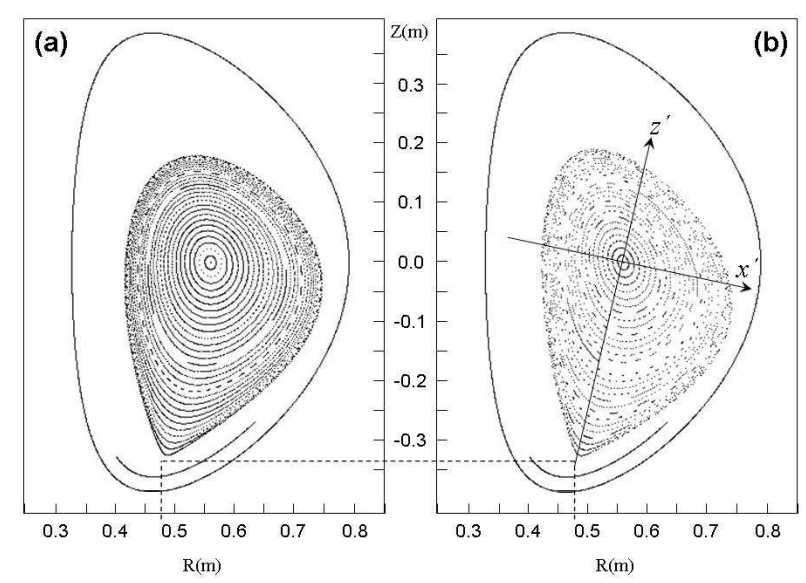

FIG. 1: $\mathbf{M}_{D}-$ generated poloidal cross sectional view of 30 single null magnetic surfaces followed up for 200 toroidal revolutions under typical operational values of COMPASS-D tokamak. The safety factor values at the magnetic axis are: (a) 1.2 and (b) 0.6, showing the two coordinate frames $\left(R=x+R_{0}, Z\right)$ and $\left(x \prime, z^{\prime}\right)$, the latter aligned with the OX segment. The single null $\mathrm{X}$ point is located with segmented lines at $R=0.47712 \mathrm{~m}, Z=-0.33387 \mathrm{~m}$. The greater curvature on the right hand side of the surfaces results from the toroidal magnetic field being inversely proportional to the major radius. Both the wall and the target plate locations are shown.

A fundamental boundary condition to value the remaining parameters in the Hamiltonian is the location of the single null cusp, otherwise known as $\mathrm{X}$ point, located at $R=R_{0}+x_{X}, z=$ $z_{X}$, whereby $\alpha=\arctan \left(x_{X} / z_{X}\right)$. On the other hand, given that the intersections of a magnetic surface with the $z$-axis take place at those $z \prime$ values which satisfy that $\gamma_{3} z^{\prime}+\gamma_{2} z^{\prime}-H=0$ for the particular $H$ value of that surface, then three real roots, 
of which two are equal, exist when the Cardan discriminant $Q \equiv\left[\left(\gamma_{2} / 3 \gamma_{3}\right)^{3}-H_{X} / 2 \gamma_{3}\right]^{2}-\left(\gamma_{2} / 3 \gamma_{3}\right)^{6}=0$ in the case of the ideal separatrix, namely, $H=H_{X}=\left(2 / \gamma_{3}\right)^{2}\left(\gamma_{2} / 3\right)^{3}$. However, we know that the Hamiltonian takes the value $H_{X}=\gamma_{3} z_{X}{ }^{3}+$ $\gamma_{2} z_{X} \prime^{2}$ on the X point and therefore $\gamma_{2}=-3 z_{X} \prime \gamma_{3} / 2$.

Two other fundamental boundary conditions can arise from the angle $\chi$ formed by the $\mathrm{X}$ point asymptotes so that $\beta_{2}=$ $R_{0}^{2} \gamma_{2} \tan ^{2}[(\pi-\chi) / 2]$. On the other hand, the Liouville theorem ensures the invariance, within any closed magnetic surface, of the amount of toroidal magnetic flux: the action variable $\psi_{T}=B_{0} R_{0} \oint_{H} z(R, H) / R d R=B_{0} R_{0} \oint_{H} z^{\prime}(\rho \prime, H) d \rho \prime$, provided that the latter integral is independent from the $\alpha$ angle. Therefore, as Fig. 1 exemplifies, by fixing a desired value of the security factor at the magnetic axis $\left(q_{0}\right)$ through $\left(\kappa B_{0} R_{0}\right)^{-1} \lim _{H \longrightarrow 0} d \psi_{T} / d H=q_{0}$, a third relationship within the parameter set $\left\{\beta_{2}, \gamma_{2}, \gamma_{3}\right\}$ allow to characterize the essential Hamiltonian for a particular device. Hence, every ideal magnetic surface being an $H=$ constant contour, the last integral can be generally evaluated as $\psi_{T}=$ $\left(2 B_{0} R_{0} / \beta_{2}\right) \int_{z_{2}^{\prime}}^{z \prime_{3}} \sqrt{H-\gamma_{3} z^{\prime}-\gamma_{2} z^{\prime 2}} d z$ where $z_{2}<0<z_{3}$ are the roots of $\rho \prime(z \prime ; H)=0$ within the confined region, namely,

$$
\begin{gathered}
z_{3}^{\prime}=\left[-\left(\gamma_{2} / \gamma_{3}\right)+(2 / d)^{1 / 3}\left(\gamma_{2} / \gamma_{3}\right)^{2}+(d / 2)^{1 / 3}\right] / 3 \\
z_{2}^{\prime}=\left[-2\left(\gamma_{2} / \gamma_{3}\right)+(2 / d)^{1 / 3}\left(\gamma_{2} / \gamma_{3}\right)^{2} i(i+\sqrt{3})+(d / 2)^{1 / 3}(-1-i \sqrt{3})\right] / 6
\end{gathered}
$$

wherever the imaginary parts of the last two complex terms cancel out each other and provided that $d \equiv-2\left(\gamma_{2} / \gamma_{3}\right)^{3}+$ $27\left(H / \gamma_{3}\right)+3 \sqrt{3 H / \gamma_{3}} \sqrt{-4\left(\gamma_{2} / \gamma_{3}\right)^{3}+27\left(H / \gamma_{3}\right)}$. Yet, as long as we are interested in the above mentioned limit, a more handy expression can be found. By realising that, near the magnetic axis, the poloidal cross sections of the magnetic surfaces approach concentric circumferences centred at the so called $\mathrm{O}$ or elliptic point (determined in the present configuration by the poloidal location of the innermost magnetic surface) then $\psi_{T} \longrightarrow \pi B_{0} R_{0} H / \beta_{2}\left|-\gamma_{2}\right|^{1 / 2}$ and therefore $\gamma_{2}=-\left(\pi / \kappa \beta_{2} q_{0}\right)^{2}$. Some typical values are: $\beta_{2}=-0.26 \mathrm{~m}$, $\gamma_{2}=-0.29 \mathrm{~m}^{-1}$ and $\gamma_{3}=-0.59 \mathrm{~m}^{-2}$.

Integrating equations (3) in the MHD ideal case is nearly trivial. In fact, defining the two functions

$$
\begin{gathered}
f\left(z^{\prime}\right) \equiv-\left(2 \gamma_{2}+3 \gamma_{3} z^{\prime}\right) z^{\prime} \\
\alpha(\rho \prime) \equiv 2 \beta_{2} \rho^{\prime}
\end{gathered}
$$

so that $z^{\prime}=\partial H / \partial \rho^{\prime}=\alpha\left(\rho^{\prime}\right)$, the Hamilton equation set can be reduced to a single second order equation of motion:

$$
\ddot{z} \prime=2 \beta_{2} \dot{\rho} \prime=-2 \beta_{2} \frac{\partial H}{\partial z^{\prime}}=2 \beta_{2} f\left(z^{\prime}\right)
$$

which admits the standard quadrature solution

$$
\tau=\frac{1}{2 \sqrt{\beta_{2} \gamma_{2}}} \int \frac{d w^{\prime}}{\sqrt{C_{1}-w^{2} \pm w^{\prime}}}+C_{2}
$$

where $w^{\prime} \equiv \pm \gamma_{3} z^{\prime} / \gamma_{2}$ and the constants $C_{1}, C_{2}$ are fixed by one boundary and one initial conditions. Thus, if $\rho /$ is considered the to be the generalised momentum, conjugate to $z$, then the generalised kinetic energy becomes

$$
\mathcal{T}=\dot{z} \prime^{2} / 2=2 \beta_{2}\left(\beta_{2} \rho \prime^{2}\right)
$$

with a generalised potential

$$
\mathcal{V}=-\int_{0}^{z \prime} \ddot{\zeta} \prime d \zeta \prime=-2 \beta_{2} \int_{0}^{z \prime} f\left(\zeta^{\prime}\right) d \zeta^{\prime}=2 \beta_{2}\left(\gamma_{2}+\gamma_{3} z^{\prime}\right) z^{\prime 2}
$$

whereby the total generalised energy is

$$
\mathcal{E}=\mathcal{T}+\mathcal{V}=2 \beta_{2} H
$$

Hence, in the absence of dissipative effects, the poloidal flux $\psi_{P}=\kappa B_{0} R_{0} H$ is a conserved surface quantity.

\section{THE STOCHASTIC SINGLE NULL TOKAMAK CONFIGURATION}

It is well known that the solutions in the previous section refer only to toroidally symmetrical ideal (i.e., non resistive) MHD stability, and this, in the absence of error fields resulting from residual magnetic coil misalignments and defects as well as internal plasma confinement degradation factors such as the presence of suprathermal particles. In any realistic study of tokamak phenomena, operational conditions have to be considered as near- or non-integrable. In the former case, such non linear marginal contributions to the magnetic field imply, in general terms, a Hamiltonian flux function transition [24]

$$
\psi_{P}=\psi_{P 0}\left(\psi_{T}\right) \longrightarrow \psi_{P}=\psi_{P 0}\left(\psi_{T}\right)+\delta \psi_{P}\left(\psi_{T}, \theta, \varphi\right)
$$

where $\theta$ is the tokamak poloidal angle. Naturally, the perturbation $\delta \psi_{P}\left(\psi_{T}, \theta, \varphi\right)$, whose specific form may not be analytically determined in full, may also not be necessarily dependent on all three coordinates. In most cases this replacement turns Hamilton's equations non separable strictly speaking [13] and yet, it is often mild enough so to allow a convenient study of the perturbed field line evolution by means of invariant preserving mappings. From a mathematical point of view, these Hamiltonian mappings are well known homeomorphisms that result from an F2 class of canonical Legendre transformations of coordinates, along the generalised time, generated by the Hamiltonian itself $[8,25]$. Therefore, a Rayleigh dissipation function with non diagonal elements excites the intrinsic stochastic attractor behaviour of 
such Hamiltonian systems, namely, in the absence of external driving forces [26]. Let us propose, then, the generalised Rayleigh dissipation potential, responsible for intrinsic magnetic flux variations, in the general form

$$
\mathcal{U}_{R}=\frac{1}{2}\left(f_{11} \dot{z} \prime^{2}+f_{12} \dot{z} \prime^{2} \dot{\rho} \prime^{2}+f_{21} \dot{\rho} \prime^{2} \dot{z} \prime^{2}+f_{22} \dot{\rho} \prime^{2}\right)
$$

where the coefficients $f_{i j}=f_{j i}, f_{i i} \neq f_{j j} ; i \neq j$. In this way, by taking derivatives with respect to the velocities, the generalised dissipation force $\mathbf{F}_{d} \equiv-\nabla_{v} \mathcal{U}_{R}$ introduces a fluctuation in the generalised energy

$$
d \mathcal{E}=-\mathbf{F}_{d} \cdot\left(\dot{\rho} \prime \hat{\rho}^{\prime}+\dot{z}^{\prime} \hat{z}^{\prime}\right) d \tau=-2 \mathcal{U}_{R} d \tau
$$

Then, given Eq. (19), the stochastic contribution to the Hamiltonian (in variational form, more convenient for our purposes) becomes

$$
\delta H=-\mathcal{U}_{R} / \beta_{2} \delta \tau
$$

which admits the solution

$$
f_{11}=f_{22}=0, \quad f_{12}=f_{21}=-\beta_{2}
$$

in order for $\delta H$ to retain its generalised energy units while following the convention of keeping $\mathcal{U}_{R}>0$ as long as $\beta_{2}<0$. Thus

$$
\delta H=\alpha(\rho) f(z \prime) \delta \tau=\dot{\rho} \prime \dot{z}^{\prime} \delta \tau .
$$

Bearing in mind that $\dot{z} \prime$ and $\dot{\rho}$ / represent the slopes of the field line's orthogonal projections with respect to the toroidal direction, then the previous equation suggests the following tokamak properties:

- Every field line with a rotational transform distinct from naught is stochastic.

- The $\mathrm{O}$ and $\mathrm{X}$ fixed points are never stochastic.

- Those magnetic surfaces closer to $z^{\prime}=$ constant planes and $\rho /=$ constant cylinders are less prone to destabilize.

- Reducing the rotational transform of the surfaces improves their stability, recouping the Kruskal-Shafranov and Mercier criteria [27] which consider the balance of toroidal and poloidal fields in terms of the amount of plasma current to be confined. That is why the reciprocal security factor central value, $1 / q_{0}$, plays a role of stochasticity parameter through the $\left\{\beta_{2}, \gamma_{2}, \gamma_{3}\right\}$ set.

A standard practice to solve the equations of motion that result from a non MHD ideal near-integrable Hamiltonian $H+\delta H$ is based on discretizing the generalised time, in our case the toroidal coordinate, so to obtain a sequence of impulsive perturbations leading to a difference equation set or map $[8,12]$. Thus, in so far as we restrict our solution, in principle, to those successive planes containing the toroidal field coils distributed with a period $\tau=\kappa \varphi / 2 \pi$, then we have effectively a perturbation

$$
\Delta H=\alpha\left(\rho^{\prime}\right) f\left(z^{\prime}\right) \delta_{\Sigma}(\tau)
$$

thanks to the periodic Dirac delta function $\delta_{\Sigma}(\tau)=$ $\sum_{k=-\infty}^{\infty} \delta(\tau-k)$. As it is frequently done, we can now introduce the 'kicked' Hamiltonian [8]

$$
H_{1}=H_{0}+\Delta H
$$

with $H_{0} \equiv H \delta_{\Sigma}(\tau)$ so that the magnetic line transition from one plane $(N)$ to the next $(N+1)$ can be expressed as

$$
\begin{gathered}
\int_{N}^{N+1} \frac{d \rho \prime}{d \tau} d \tau=-\int_{N}^{N+1} \frac{\partial H_{1}}{\partial z \prime} d \tau, \\
\int_{N}^{N+1} \frac{d z^{\prime}}{d \tau} d \tau=\int_{N}^{N+1} \frac{\partial H_{1}}{\partial \rho \prime} d \tau .
\end{gathered}
$$

Then, as $\Delta H \longrightarrow 0$, we use the unperturbed values of $\rho /, z^{\prime}$ so to integrate $\partial H_{1} / \partial z^{\prime}$ over the unperturbed orbits [8]:

$$
\rho^{\prime}{ }_{N+1}=\rho^{\prime}{ }_{N}+f\left(z_{N}^{\prime}\right), \quad z^{\prime}{ }_{N+1}=z_{N}^{\prime}+\alpha\left(\rho \prime_{N}\right)+2 \beta_{2} f\left(z_{N}^{\prime}\right)
$$

The latter mapping is not fully invariant preserving, though. It does not preserve the poloidal flux given the fluctuation $\delta \psi_{P}=\kappa B_{0} R_{0} \delta H$ where $\delta H$, understood as the product of the field line slopes as in Eq. (25), is not zero in general and so $\psi_{P}$ is not a surface quantity. Nevertheless, the previous mapping does preserve the toroidal flux provided that the Jacobian determinant

$$
|\mathcal{I}|=\left|\partial\left(\rho^{\prime}{ }_{N+1}, z_{N+1}^{\prime}\right) / \partial\left(\rho_{N}, z_{N}\right)\right|=1
$$

A more straightforward way, although far less conventional, to obtain Eqs. (29) from Eqs. (3) provided that constraint Eq. (30) is satisfied, can be described as follows. Clearly, from the definition of total derivative, if $\tau_{0}$ is a constant,

$$
\begin{aligned}
& \lim _{\delta \tau \longrightarrow 0} \frac{z^{\prime}\left(\tau_{0}+\delta \tau\right)-z^{\prime}\left(\tau_{0}\right)}{\delta \tau}=\left[\frac{\partial H}{\partial \rho^{\prime}}\right]_{\tau=\tau_{0}}, \\
& \lim _{\delta \tau \longrightarrow 0} \frac{\rho \prime\left(\tau_{0}+\delta \tau\right)-\rho \prime\left(\tau_{0}\right)}{\delta \tau}=-\left[\frac{\partial H}{\partial z^{\prime}}\right]_{\tau=\tau_{0}}
\end{aligned}
$$

As defined, $\tau=\kappa \varphi / 2 \pi$ so that, by considering the limit $\delta \tau \longrightarrow$ 1 instead, a measure of the field line shift after one $\kappa$-th of toroidal revolution can be obtained as

$$
\begin{gathered}
z^{\prime}{ }_{N+1}-z_{N}^{\prime}=\left[\frac{d z^{\prime}}{d \tau}\right]_{\tau=\tau_{a}}=\left[\frac{\partial H}{\partial \rho \prime}\right]_{\tau=\tau_{a}}, \\
\rho^{\prime}{ }_{N+1}-\rho \prime_{N}=\left[\frac{d \rho \prime}{d \tau}\right]_{\tau=\tau_{b}}=-\left[\frac{\partial H}{\partial \rho \prime}\right]_{\tau=\tau_{b}}
\end{gathered}
$$

where the $N, N+1$ subindices refer to $\tau_{0}$ and $\tau_{0}+1$, respectively, while $\tau_{a}, \tau_{b}$ are two values of $\tau$ between $\tau_{0}$ and $\tau_{0}+1$ according to the Mean Value Theorem. Imposing no other constraint than the canonical one given by Eq. (30), one obtains the solutions

$$
\tau_{a}=\lim _{\tau \longrightarrow \tau_{0}+1} \tau, \quad \tau_{b}=\lim _{\tau \longrightarrow \tau_{0}} \tau
$$


whereby Eqs. (32) become Eqs. (29). They can be reexpressed in the also more compact matrix operator notation

$$
\left(\begin{array}{c}
\rho \prime_{N+1} \\
z \prime_{N+1}
\end{array}\right)=\mathbf{M}_{D}\left(\begin{array}{c}
\rho \prime_{N} \\
z \prime_{N}
\end{array}\right)
$$

thanks to the map operator (the symbol $\circ$ indicates functional composition):

$$
\mathbf{M}_{D} \equiv\left(\begin{array}{cc}
1 & \zeta_{\circ} \\
2 \beta_{2} & 1+2 \beta_{2} \zeta_{\circ}
\end{array}\right)
$$

satisfying naturally that $\left|\mathbf{M}_{D}\right|=1$ and where we have defined the distributive functional operation $\zeta \circ \equiv-\left(2 \gamma_{2} \mathbf{I}+3 \gamma_{3} \mathbf{I}^{2}\right) \circ$ so that $\mathbf{I}^{n} \circ x=x^{n}$ for any real $x$ and any integer $n$.

By lowering the level of the $q$-profile by means of $q_{0}$ in $\mathbf{M}_{D}$ so to approach the value $q=1$ at the edge, an increasing number of regular magnetic surfaces destabilize creating bands of stochasticity limited by some more stable (less $q$-rational) surfaces, in a process predicted by the KruskalShafranov-Mercier criterium [27] and described by the KAM Theorem [8]. Henceforth, a magnetic surface cross section portrait of the COMPASS-D tokamak scenario is presented in Fig. 1 for the cases $q_{0}=1.2$ and $q_{0}=0.6$, so to cover the above mentioned transition. One toroidal revolution corresponds to the transformation $\mathbf{M}_{D}^{\mathrm{k}}$. As expected, the contours are not symmetric with respect to the $z$ axis given that the stabilizing toroidal field decays with $1 / R$.

\section{ON THE TOPOLOGY OF THE STOCHASTIC SEPARATRIX}

It is not unfrequent that purely numerical modelling produces some artificial ergodization near the X-point. It might even be suspected that the stochasticity discussed in the previous section is due to mere numerical inaccuracies. One of the characteristics that make canonical, namely measure preserving, mappings like $\mathbf{M}_{D}$ so powerful is precisely that the numerical diffusion resulting from roundoff error occurs along the magnetic surfaces and not across them [28]. On the contrary, it is well known that actual magnetic field lines from destabilizing surfaces can move transversely to them and may migrate filamentary or remain stationary in island chains and/or other subsidiary structures but, fortunately to plasma confinement, cannot penetrate regular, less $q$-rational, surfaces. Mappings like $\mathbf{M}_{D}$ share such behaviour, stipulated in the KAM theorem: transverse diffusion depends on local rationality and not on global roundoff error. This theorem, conjectured by A.N. Kolmogorov and J. Moser and later on very formally demonstrated by V.I. Arnold [29], applies to Hamiltonian systems in the phase space which, in the case of tokamaks, happen to coincide practically with the physical space. Thus, provided that an unperturbed Hamiltonian satisfies some conditions of non resonance [8], many of the unperturbed toroidal magnetic surfaces are preserved in the semi integrable case, namely, for mild perturbations. These so called KAM surfaces are important because they limit the stochastic regions preventing the magnetic field from wandering throughout the space and so helping the plasma confinement. Furthermore, the stability condition of the KAM theorem with respect to perturbation can be expressed as a diophantine form [30] whereby the ratio of the toroidal and poloidal revolutions of a magnetic line determines the stability of the toroidal surface it weaves. Highly rational line ratios, in the sense of a continued fraction expansion as in [31], are structurally unstable. Less rational lines which, however, do not satisfy the diophantine condition, end up wandering stochastically only after having followed apparently stable trajectories. The more irrational cases, diophantine stable, become KAM invariant torii. Of them, the last to succumb is, famously, the one corresponding to the golden mean: $(\sqrt{5}-1) / 2$.

The mapping $\mathbf{M}_{D}$ and its inverse $\mathbf{M}_{D}^{-1}$ enables us to explore the edge topology by considering a row of equidistant initial points such as $\left\{\mathbf{x}_{\mathbf{0}}=\mathbf{x}(R, z=0, \varphi=0) \mid R_{\text {lcfs }}<R<\right.$ $\left.R_{l c f s}+\lambda_{\text {sol }}\right\}$ where $R_{l c f s}$ is the position of the outmost close flux surface on the outboard midplane and $\lambda_{\text {sol }}$ is a typical width of the scrape off layer which can be defined as the portion of the open surface region that is effectively occupied by plasma particles. The successive crossings of each field line trajectory through the $\varphi=0$ plane may be registered on a slab geometry plane $\psi_{T}$ vs $\theta$ (or rather, for the sake of clarity, $R\left(\psi_{T}\right)$ vs $\theta$ as in Fig. 2) where $\theta=\arctan \left[z /\left(R-R_{0}\right)\right]$ is the poloidal angle that starts from the outboard midplane. The surface quantity of magnetic toroidal flux, $\psi_{T}$, is measured as the value $\oint_{C} \mathbf{B} \cdot \hat{\varphi} d s$ on the poloidal plane between the magnetic axis and the contour $\mathcal{C}$ generated by the crossings, as long as field line remains within the wall. Should $C$ be a connected contour then the value of the integral is normalised by the number of poloidal revolutions. In this context, the following hierarchy can be established.

- Scrape Off Layer (SOL): where the magnetic field lines exit the nested flux surface region after half a poloidal revolution. Its width is conventionally determined by the presence of a limiter or by conditions like the value of the decay constant of the midplane power flux distribution functions, depending on the local particle diffusion across the magnetic surfaces.

- Stochastic Layer: where the lines exit the nested flux surface region after one or more poloidal revolutions. This layer, together with the previous one, are often known as the loss region.

- Close Surface Region: where the lines exit the nested flux surface region after a conventionally 'infinite' number of poloidal revolutions. Such number can be established on the basis of criteria like the confinement times.

The fact that the number of poloidal revolutions exhibits the tendency to diminish outwards (one common characteristic to many purely numerical non ideal tokamak field line tracing results [32]) does not necessarily imply that it occurs smoothly or even monotonically. In the transitional stochastic layer, very long lines seem to coexist next to lines that break away after a very few transits, clearly due to their contrasting rationality. Thus, Fig. 3 exhibits a $\mathbf{M}_{D}$-generated spiky 
(a)

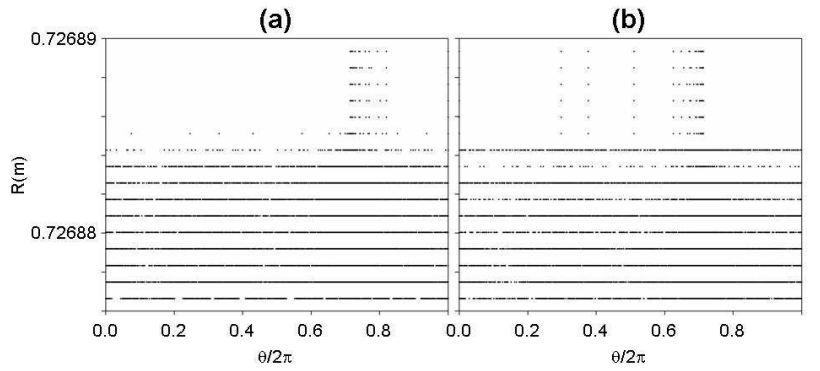

FIG. 2: Poloidal cross sectional view of the stochastic separatrix on the $R\left(\psi_{T}\right)$ vs $\theta$ plane. The normalized poloidal angle $\theta$ value starts on the outboard midplane while $\psi_{T}$ labels the surfaces, increasing with the minor radius. $R\left(\psi_{T}\right)$ is the starting major radial location of the $\psi_{T}$-surface, also on the outboard midplane. Sixteen initial conditions, launched from $\theta=0$, are followed up by (a) $\mathbf{M}_{D}$ and (b) $\mathbf{M}_{D}^{-1}$. In both cases, the closer the field lines are to the $\mathrm{X}$ point the 'slower' their poloidal progress becomes until the outmost lines fail to resume it and end up on the target.

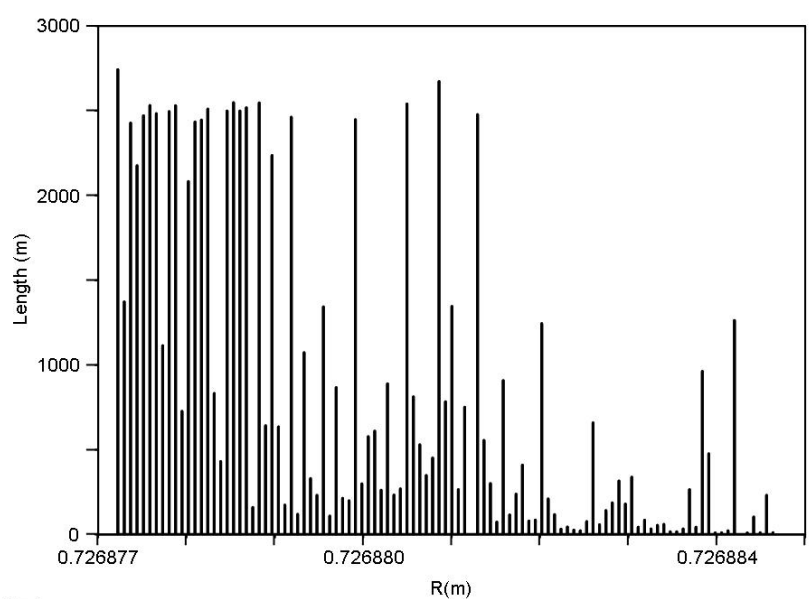

FIG. 3: Length vs launching position on the outboard midplane of magnetic field lines within the stochastic separatrix band, when $q_{0}=0.6$. This $\mathbf{M}_{D}-$ generated spectrum is limited to 250 poloidal transits. The field lines are followed until they wander away from the column and touch the wall. The uneven length distribution suggests the connection between stability and safety factor rationality.

field line length spectrum collapsing into a less bristly declining pattern once the open surface region, the scrape off layer (SOL)[1] has been reached where the mostly parallel transport of particles and energy to the target takes place.

Although Fig. 2 suggests a reluctant poloidal progress of the field line when approaching the magnetic cusp $\mathrm{X}$ point, a more revealing insight of the plasma edge is gained by focusing on that neighbourhood in $\left(R, z, \psi_{T}\right)$-coordinates as shown in Fig. 4. Under MHD ideal conditions, the helical pitch of the field lines vanishes throughout the infinitely thin separatrix so to avoid either splitting one ingoing line into two outgoing ones at the $\mathrm{X}$ point or else creating a sink there. On the other hand, the near integrable stochastic cusp is a fixed point of the Hamiltonian with significant consequences: the poloidal com-

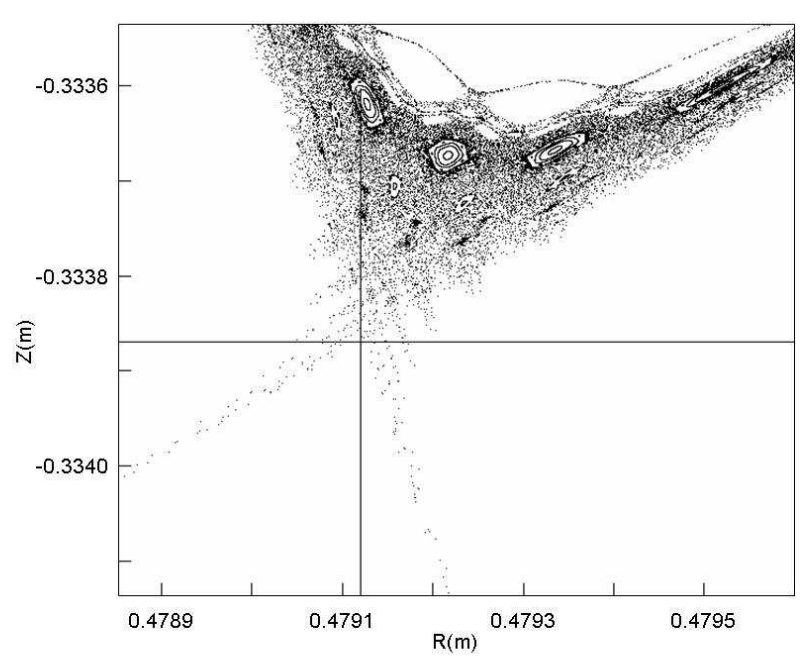

FIG. 4: A $1500 \times$ amplification of the $\mathbf{M}_{D}^{ \pm \mathrm{K}}$-generated stochastic separatrix centered on the single cusp, showing the genesis of the typical deposition patterns on the target plate. One thousand outer surfaces have been followed up to 500 poloidal revolutions when $q_{0}=0.7$ for stochasticity enhancement, starting from a last confined surface located, on the outboard midplane, at $R=0.731043 \mathrm{~m}$. The magnetic surfaces become increasingly ruffled as approaching the $\mathrm{X}$ point to the extent of projecting themselves onto the target, propitiating the particle flux given the higher parallel conductivity.

(a)

(b)

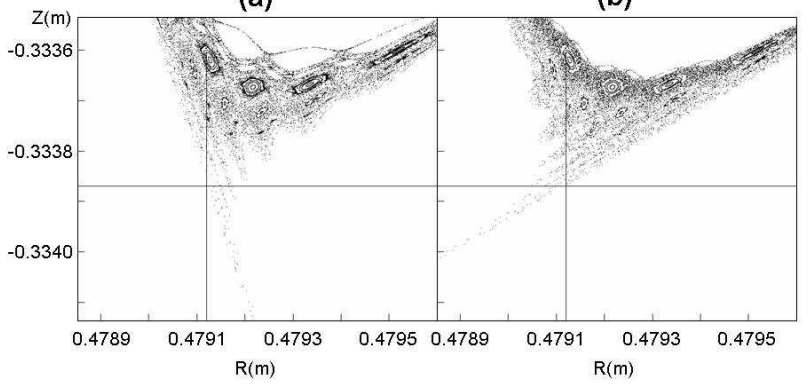

FIG. 5: (a) $\mathbf{M}_{D}^{+\kappa}-$ component of the previous figure as initial points on the outboard midplane are followed clockwise. (b) $\mathbf{M}_{D}^{-\mathrm{K}}$-component following the lines counterclockwise. The superimposition of both trajectory sets results in a trellis structure, characteristic of the stochastic cusp region.

ponent of the lines fades away as the $\mathrm{X}$ point is approached so to ensure that the line progress there is purely toroidal. Thus, a line of the stochastic layer approaches the OX segment undergoing a steady reduction in its helical pitch but, given that $\nabla \cdot \mathbf{B}=0$, then the outmost lines avoid stagnation by undertaking increasingly pronounced excursions [33] as shown in Fig. 4. Yet, such undulation might introduce either an excess or a deficit in the poloidal area, namely the toroidal flux as we have previously discussed, contained by the magnetic surface. This would contradict the Liouville Theorem, so that the excursion is immediately redressed only to lapse into spikier ripple. Although, in the limit, this resonant behaviour gives 
rise to the private region legs of the separatrix, the lines oscillate transversally to the poloidal circulation sense just to reassume the nested geometry [5]. Furthermore, the edge line evolution bifurcates increasingly outwards making the trajectories of the direct $\mathbf{M}_{D}^{+\kappa}$-branch complementary to those of the inverse $\mathbf{M}_{D}^{-\kappa}$-branch, as exhibited in Fig. 5. More explicitly, it portrays how the edge lines escape the confinement region after a finite number of poloidal revolutions, either on the outboard or the inboard side according to their left (right) handedness with respect to the toroidal coordinate. That is why, if one same toroidal sense is maintained by the observer, then every escaping line appears to resume its poloidal circulation after an infinitely long excursion. And yet, both branches of one same magnetic surface can intersect each other at points known in topology as homoclinic (Fig. 4). As the edge is approached, the rather explosive oscillatory topology of the surfaces can clearly develop to the extent of touching the surface of the target plate with considerable repercussions as to the transport of power and particles given the greater parallel conductivity. In this way, the general deposition pattern is expected to adopt the well known parallel band pattern that runs toroidally on the target with a wider distance among such rings in the case of the outboard side of the separatrix.

\section{CONCLUSIONS}

It has been shown that a compact and yet rich mapping of the tokamak divertor magnetic configuration can be devel- oped consistently from the Hamiltonian function itself. Although this model shares with previous attempts by several authors many of the advantages of canonical maps such as a very fast processing and the absence of error propagation, in our case no heuristic adaptation of alien maps or geometries has been required. It follows a considerable proximity between a device and its model of which the upholding of the Kruskal-Shafranov-Mercier criterion is a significant instance. Likewise, the intricate stochastic separatrix revealed by the $\mathbf{M}_{D}$ map culminates at the neighbourhood of the $\mathrm{X}$ point cusp. There, the transverse flexion of of the adjoining magnetic surfaces follows directly from the Liouville invariance of the toroidal flux. The mutually complementary crisscrossed topology of the $\mathbf{M}_{D}^{+\kappa}$ and $\mathbf{M}_{D}^{-\kappa}$ branches of the separatrix combines with the higher parallel conductivity to create the fairly universal toroidally annular deposition pattern on the target plate.

\section{Acknowledgements}

The authors are especially grateful to Professors E. Piña and L. Jimenez-Lara for valuable observations and referential support.
[1] P.C. Stangeby and G.M. McCracken, Nucl. Fusion 30, 1225 (1990).

[2] Ph. Ghendrich, Plasma Phys. Control Fusion 34, 2007 (1992).

[3] F. Ryter et al., Plasma Phys. Control Fusion 38, 1279 (1996).

[4] J.A. Goetz et al., Phys. Plasmas 3, 1908 (1996).

[5] T.E. Evans, R.K.W. Roeder et al. Contrib. Plasma Phys. 44 1-3, 235 (2004).

[6] A. Boozer, Phys. Fluids 26, 1288 (1983).

[7] B. V. Chirikov, Atomnaya energya 6, 630 (1959).

[8] A.M. Lichtenberg and M.A. Libermann, Regular and Stochastic Motion 26 Springler Verlag, 1983

[9] F.A. Haas and A. Thyagaraja, Phys. Rep. 134(4), 240 (1986).

[10] R.B. White and Y. Wu, Plasma Phys. Control. Fusion 30, 183 (1993).

[11] A. Punjabi, H. Ali, and A. Boozer, Phys. Plasmas 4 (2) (1997) 33.

[12] E.C. da Silva, I.L. Caldas, and R.L. Viana, Braz. J. Phys. 32 (1), 39 (2002).

[13] R. Balescu, M. Vlad, and F. Spineanu, Phys. Rev. E 58(1), 951 (1998).

[14] B. V. Chirikov, Phys. Rep. 52, 265 (1979).

[15] R. Balescu, Phys. Rev. E 58(3), 3781 (1998).

[16] R.H. Rebut and M. Hugon, Plasma Phys. Control. Fusion Research 2, 187 (1985).

[17] A. Punjabi, A. Verma, and A. Boozer, Phys. Rev. Lett. 69 (23), 3322 (1992).

[18] A. Punjabi, H. Ali, and A. Boozer, Phys. Plasmas 10 (10), 3992 (2003).
[19] United Kingdom Atomic Energy Authority. The COMPASS Project Safety Assessment Document Culham Laboratory (1989)

[20] R.T. Crossland, Proc. 14th SOFT 2 (1986) 1781.

[21] C. L1. Thomas, Report CLM-R133, Culham Laboratory, 1979.

[22] A.B. Rechester, M.N. Rosenbluth, Phys. Rev. Lett. 40 (1), 38 (1978).

[23] A. Coté, et al. 1985 European Conference Abstracts Proc. 12th European Conference on Controlled Fusion and Plasma Physics. Part II, p. 450

[24] S.S. Abdullaev, K.H. Finken, and A. Kaleck, Phys. Plasmas 5 (1), 196 (1998).

[25] H. Goldstein, Classical Mechanics 2nd Ed. Chapter 9. Addison Wesley Publishing Company, 1980

[26] V. Sheeja, M. Sabir, Int. J. Bifurcation and Chaos 12 (4) (2002) 859.

[27] R.B. White, The Theory of Toroidally Confined Plasmas 2nd Ed. Imperial College Press, 2001

[28] J.M. Green, J. Math. Phys. 20 (6), 1183 (1979).

[29] V.I. Arnold, Sov. Math. Dokl. 3, 136 (1963).

[30] J. Moser, Stable and random motions Princeton University Press, 1973

[31] A. Ya. Khinchin, Continued Fractions Chicago University Press, 1964.

[32] N. Pomphrey, A. Reiman, Phys. Fluids B 4 (4), 938 (1992).

[33] F. Nguyen et al., J. Nucl. Mater. 176-177, 499 (1990). 